\title{
A Clinical Study of Psychiatric Morbidity and Quality of Life among Suicide Attempted Patients
}

\author{
Dr. Challa Venkata Suresh ${ }^{1 *}$
}

\section{ABSTRACT}

Risk of suicide is more common in patients with psychiatric disorders and poor quality of life. The aim of this study was to find out the prevalence of psychiatric morbidity and its relationship with suicide attempters. Materials and Method- The cross sectional study was carried out in Psychiatric department of MNR Medical College and Hospital. A total 70 cases of first suicide attempts were included in this study. Psychiatric morbidity and quality of life were analysed by Schedule for clinical assessment in neuropsychiatric (SCAN), Montgomery Asberg's depression rating scale (MADRS), Hamilton anxiety rating scale (HAM-A) and WHOQOL-BREF version. Statistical analysis was done by SPSS 20.0 software. Result- Among 70 cases, 92.85\% of the suicide attempters had one or more psychiatric disorders compared to $21.43 \%$ among the controls. Major psychotic disorder was mood disorder (56.92\%), followed by Neurotic and somatoform disorders (36.92\%) and substance related disorders (32.3\%). Schizophrenia and other psychotic disorders were diagnosed in 7.69\% of cases. Quality of life score was assessed by WHOQOL-BREF questionnaire. The mean of total score in suicide attempters was 57.44 and in control group was 73.67. Conclusion- In the present study suicide attempters had higher psychotic morbidity and poor quality of life in comparison to the control groups.

Keywords: Psychiatric Disorders, Quality Of Life, Attempted Suicide.

Suicidal tendency refers to a self-destructive behaviour, myriad of cognition specific to death (Pfeffer CR et al (1991), Smith K and Crawford S (1986), Kessler RC (1999). According to WHO suicide attempt as "any act arising from a deliberate wish of self-destruction, no matter whether the intention to die was strong, ambivalent or vague” (World Health Organization (1992). National Crime Records Bureau (NCRB) report shows national average suicide rate of 11 in 100,000 and suicide rates vary across the different state of India (National Crime Records Bureau (2014). Mental disorders such as depression, anxiety, bipolar disorder, schizophrenia and alcohol/substance abuse etc. can increase the risk of suicidal behaviour in persons (World Health

\footnotetext{
${ }^{1}$ Professor \& HOD Department of Psychiatry, MNR Medical College, Fasalwadi, Sangareddy, Telangana State, India

*Responding Author

(C) 2016 C Suresh; licensee IJIP. This is an Open Access Research distributed under the terms of the Creative Commons Attribution License (http://creativecommons.org/licenses/by/2.0), which permits unrestricted use, distribution, and reproduction in any Medium, provided the original work is properly cited.
} 


\section{A Clinical Study of Psychiatric Morbidity and Quality of Life among Suicide Attempted Patients}

Organization (2001).Risk factors for suicidal behaviour are more complex and interacting with each other. Mood disorders and schizophrenia are the most common cause of suicide among all other risk factors (Sartorius N. et al (1986). Thus the present study was aimed to find out the prevalence rate and relationship between psychiatric disorders and quality of life among suicide attempters.

\section{MATERIAL AND METHODS}

A cross-sectional study was carried out in Psychiatric department, MNR Medical College and Hospital, Sangareddy. Institutional ethical committee approved the study. 70 cases of first suicide attempt were included in this study and all the cases were compared with 70 controls selected randomly from general population. The informed consent form was taken from all the patients. The personal data of all patients were recorded in well-designed information sheet. Psychiatric assessment was done according to SCAN based interview. Using MADRS assessed level of depression and HAM-A was used for assessing level of anxiety. WHOQOL-BREF was used to assess quality of life among suicide attempters and controls subject. All data was analysed by SPSS 20.0 software. Chi-Square test and t-test were performed to analyse the data.

\section{RESULT}

Among 70 cases, 55 (78.57\%) were male and 15 (21.43\%) were female patients. 92.85\% of the suicide attempters had one or more psychiatric disorders compared to $21.43 \%$ among the controls. The difference was statistically significant $(\mathrm{P}<0.05)$. Major psychotic disorder was mood disorder (56.92\%), followed by Neurotic and somatoform disorders (36.92\%) and substance related disorders (32.3\%). Schizophrenia and other psychotic disorders were diagnosed in $7.69 \%$ of cases (Table1). Quality of life score was assessed by WHOQOL-BREF questionnaire. The mean score of physical, psychological, social and environmental domain in study group was 54.32, 53.54, 65.34, and 56.56 while in control group score was 76.45, 73.26, 77.65, 67.32 respectively. The mean of total score in suicide attempters was57.44 and in control group was 73.67 (Table2). So the data revealed the score was higher in control group than study group and the difference was statistically significant. Thus the quality of life among control group was significantly better.

\section{DISCUSSION}

Psychological disorders are the most common risk factors in suicidal cases. Robins E et al. study revealed that suicide dose not occur absence of mental illness and alcoholism (Robins $\mathrm{E}$ et al (1959). In this present study 92.85\% suicide attempters was found with multiple psychological disorders as compared to control group (21.43\%). Our findings were comparable with other studies done by Sharma RC (1998) and Sameer Saharan et al. (2014). The most common diagnosis was mood disorder (56.92\%), neurotic and somatoform disorders (36.92\%) and substance related disorders (32.3\%). 7.69\% cases were diagnosed with schizophrenia and other psychotic disorders. Sameer Saharan et al. reported most common psychological disorder was 


\section{A Clinical Study of Psychiatric Morbidity and Quality of Life among Suicide Attempted Patients}

mood disorder (52\%), adjustment disorder (26\%) and substance related disorder (28\%) (Sameer Saharan et al. (2014). Klerman GL study showed anxiety disorder, somatoform disorder, panic disorder was not associated with increased risk of suicide (Klerman GL (1987). Neurotic and somatoform disorders (36.92\%) were second most common psychiatric morbidity in present study. Other studies showed that adjustment disorder was more common than neurotic and somatoform disorders (Sameer Saharan et al. (2014), Chiu LW (1989), Kumar PNS (1998), Kumar PNS (1995). Substance related disorders (32.3\%) were the third most common risk factors in suicide attempters. Our findings were similar to other study reports (Sameer Saharan et al. (2014), Angst J et al (1998), Oquendo MA (2006). In this current study, 21.43\% control group was diagnosed with psychotic disorder. Majority of them were male than female, who suffered from psychological disorder. Nordentoft $\mathrm{M}$ et al. reported prevalence of psychotic disorder was $21 \%$ in control group and comparatively lower psychological disorder (13\%) rate was found in another study (Nordentoft M et al (1993), Sameer Saharan et al. (2014). In this present study 7.69\% cases had schizophrenia and other psychotic disorders. But Verona et al reported schizophrenia (46.9\%) and bipolar disorder (55.6\%) are more common in suicide attempters (Verona E et al (2004). Sato et al and Srivastava et al reported low prevalence rate of psychotic disorder associated with suicide, $16.3 \%$ and $11.6 \%$ respectively (Sato T et al (1993),Srivastava MK et al (2004). In this current study quality of life was significantly higher among control group (73.67\%) than suicide attempters (57.44\%). Various study reported suicide attempters had poor quality of life (Sameer Saharan et al. (2014),Phillips MR et al (2002), Cui S et al (2003),Wang S et al (2013).

In this case control study we found out that suicide attempters had higher psychiatric disorders such as depression, neurotic and somatoform disorders and substance related disorders. Poor quality of life was found out in suicide attempters as compared to control group. Therefore it is useful to assess the psychiatric morbidity and quality of life among suicide attempters, which can help us to provide proper counselling, psychotherapy and pharmacotherapy to the patients.

\section{Acknowledgements}

The author appreciates all those who participated in the study and helped to facilitate the research process.

Conflict of interest: None declared

\section{REFERENCES}

Angst J, Clayton PJ (1998). Personality, smoking and suicide: a prospective study. J Affect Disord, 51: 55-62.

Chiu LW (1989). Attempted suicide in Hong Kong. Acta Psychiatr, 79: 425-30.

Cui S, Yang R, He F, Zheng Y (2003). Case-control analysis and follow up study on risk factors of suicide attempt in a rural population in Shandong Province. Wei Sheng Yan Jiu, 32: 562-4. 


\section{A Clinical Study of Psychiatric Morbidity and Quality of Life among Suicide Attempted Patients}

Kessler RC, Borges G, Walters EE (1999). "Prevalence of and risk factors for lifetime suicide attempts in the National Comorbidity Survey”. Archives of General Psychiatry, 56:617-626.

Klerman GL (1987). Clinical epidemiology of suicide. J Clin Psychiatry, 48(12): 33-8.

Kumar PNS (1998). Age and gender related analysis of psychosocial factors in attempted suicide. Indian J Psychiatry, 40(4): 338-45.

Kumar PNS, Kuruvilla K, Dutta S, John G. Jayaseelan (1995). Psychological aspects of attempted suicide: study from a medical intensive care unit. Indian J Psychological Medicine, 18(2): 32-42.

National Crime Records Bureau (2014). Accidental Deaths and Suicides in India 2013 (ADSI2013). Ministry of Home Affairs, Government of India New Delhi.

Nordentoft M, Rubin P (1993). Mental illness and social integration among suicide attempters in Copenhagen: comparison with the general population and a four-year follow-up study of 100 patients. Acta Psychiatr Scand, 88: 278-85.

Oquendo MA, Currier D, Mann JJ. (2006), "Prospective studies of suicidal behaviour in major depressive and bipolar disorders: what is the evidence for predictive risk factors?” Acta Psychiatr Scand, 114: 151-8.

Pfeffer CR, Klemm GL, Hurt SW, et.al. (1991) "Suicidal children grow up: Demographic and clinical risk factors for adolescent suicide attempts”. Journal of the American Academy of Child and Adolescent Psychiatry, 30: 609-616.

Phillips MR, Yang G, Zhang Y, Wang L, Ji H, Zhou M (2002). Risk factors for suicide in China: A national case control psychological autopsy study. Lancet, 360: 1728-36.

Robins E, Murphy GE, Wilkinson RH, Gassner S, Kayes J. (1959) Some clinical considerations in the prevention of suicide based on a study of 134 successful suicides. Am J Publ Health, 49: 888-99.

Sameer Saharan, ManjuBhaskar (2014). Psychiatric Morbidity and Quality of Life in Suicide Attempters. J of Evolution of Med and Dent Sci. 54(3): 12438-12447.

Sartorius N. et al (1986). "Early Manifestations and First Contact Incidence of Schizophrenia in Different Cultures”. Psychological Medicine, 16: 909-928.

Sato T, Takeichi M, Hara T (1993). Suicide attempts by agricultural chemicals. Indian J Psychiatry, 35(4): 209-10.

Sharma RC (1998). Attempted suicide in Himachal Pradesh. Indian J Psychiatry, 40(1): 50-4.

Smith K and Crawford S (1986). Suicidal behaviour among "normal” high school students. Suicide and Life-Threatening Behaviour, 16: 313-325.

Srivastava MK, Sahoo RN, Ghotekar LH, Dutta S, Danabalan M, Dutta TK et al. (2004), "Risk factors associated with attempted suicide: a case control study”. Indian J Psychiatry.2004; 46(1): 33-8.

Verona E, Sachs-Ericsson N, Joiner Jr. TE (2004). Suicide attempts associated with externalizing psychopathology in an epidemiological sample. Am J Psychiatry, 161(3): 444-51. 
Wang S, Chou Y, Yeh M, Chen C and Tzeng W (2013). Factors associated with quality of life after attempted suicide: a cross sectional study. Journal of Clinical Nursing.doi: 10.1111/jcon.12148.

World Health Organization (1992).International Classification of Diseases. Manual of the International Statistical Classification of Diseases: Injuries and Causes of Deaths. $10^{\text {th }}$ revision. Geneva, World Health Organization.

World Health Organization (2001).“ Mental and Neurological Disorders”. Fact sheet No. 265.www.who.int.

Table 1: Psychiatric disorders among suicide attempters and control group $(P<0.05)$

\begin{tabular}{|l|c|c|}
\hline Psychiatric Disorders & Suicide attempters (N= 65) & Control group (N= 15) \\
\hline Mood disorders & 37 & 5 \\
\hline $\begin{array}{l}\text { Schizophrenia and other } \\
\text { psychotic disorders }\end{array}$ & 5 & 0 \\
\hline $\begin{array}{l}\text { Neurotic and somatoform } \\
\text { disorders }\end{array}$ & 24 & 10 \\
\hline Substance related disorders & 21 & 8 \\
\hline
\end{tabular}

$\mathrm{X}^{2}=83.5 ; \mathrm{df}=1 ; \mathrm{P}<0.05$ Significant

Table 2: Quality of Life among suicide attempters and control group (WHOQOL-BREF Scale)

\begin{tabular}{|l|l|l|l|l|}
\hline Quality of Life & $\begin{array}{l}\text { Suicide } \\
\text { attempters }\end{array}$ & Control group & t-test score & P value \\
\hline $\begin{array}{l}\text { Physical health } \\
\text { and well being }\end{array}$ & $54.32+14.14$ & $76.45+8.96$ & 12.5167 & 0.0001 \\
\hline $\begin{array}{l}\text { Psychological } \\
\text { health and well } \\
\text { being }\end{array}$ & $53.54+15.75$ & $73.26+12.85$ & 9.1268 & 0.0001 \\
\hline Social relations & $65.34+15.20$ & $77.65+12.88$ & 6.1538 & 0.0001 \\
\hline Environmental & $56.56+16.45$ & $67.32+12.27$ & 5.7209 & 0.0001 \\
\hline Total & $57.44+15.38$ & $73.67+11.74$ & 8.3795 & 0.0001 \\
\hline
\end{tabular}

How to cite this article: C Suresh (2016), A Clinical Study of Psychiatric Morbidity and Quality of Life among Suicide Attempted Patients, International Journal of Indian Psychology, Volume 3, Issue 4, No. 77, ISSN:2348-5396 (e), ISSN:2349-3429 (p), DIP:18.01.063/20160304, ISBN: 978-1-365-51571-2 